\title{
Association Between Plasma Glycosylated Hemoglobin A1c/high-density Lipoprotein Cholesterol Ratio and Carotid Atherosclerosis: A Real-world Study
}

\section{Xiangming Hu}

Southern Medical University

Wei Li

Guangdong Provincial People's Hospital

Chenyang Wang

Guangdong Provincial People's Hospital

Haotian Zhang

Guangdong Provincial People's Hospital

Haoyu Lu

Guangdong Provincial People's Hospital

Guang Li

Guangdong Provincial People's Hospital

\section{Yingling Zhou}

Guangdong Provincial People's Hospital

haojian dong ( $\nabla$ donghaojian@sina.com )

Guangdong Provincial People's Hospital https://orcid.org/0000-0002-4388-8623

\section{Original investigation}

Keywords: atherosclerosis, glycosylated hemoglobin A1c, high-density lipoprotein cholesterol, carotid artery intima-media thickness, carotid artery plaque

Posted Date: July 26th, 2021

DOl: https://doi.org/10.21203/rs.3.rs-724546/v1

License: (1) This work is licensed under a Creative Commons Attribution 4.0 International License. Read Full License 


\section{Abstract}

Background: Diabetes mellitus (DM) and dyslipidemia are the main risk factors for atherosclerosis. Elevated glycosylated hemoglobin A1c (HbA1c) and reduced high-density lipoprotein cholesterol (HDL-C) are associated with the progression of atherosclerosis. The aim of this study is to explore the relationship between the $\mathrm{HbA} 1 \mathrm{c} / \mathrm{HDL}-\mathrm{C}$ ratio and atherosclerosis evaluated using carotid artery intima-media thickness (cIMT) and carotid artery plaque.

Methods: In this retrospective study, we enrolled 1304 patients who had multiple cardiovascular risk factors or symptoms of suspected coronary artery disease. cIMT and carotid artery plaque were measured using ultrasonography. Logistic regression was used to explore the correlation between the $\mathrm{HbA} 1 \mathrm{c} / \mathrm{HDL}-\mathrm{C}$ ratio and cIMT or carotid artery plaque. We used restricted cubic spline curves to assess nonlinear relationships between the $\mathrm{HbA} 1 \mathrm{c} / \mathrm{HDL}-\mathrm{C}$ ratio and cIMT or carotid artery plaque.

Results: With increased quartiles of $\mathrm{HbA} 1 \mathrm{c} / \mathrm{HDL}-\mathrm{C}$, patients had higher cIMT and a greater carotid plaque burden. After adjusting for other relevant clinical covariates, patients with the highest $\mathrm{HbA} 1 \mathrm{c} / \mathrm{HDL}-\mathrm{C}$ ratio (Quartile 4 [Q4]) had a 2.88-times (95\% confidence interval [CI]: 2.02-4.10, $\mathrm{P}<0.001$ ) more abnormal mean cIMT, 3.72-times (95\% Cl: 2.55-5.44, P<0.001) more abnormal maximum clMT, and 2.58-times (95\% Cl: $1.70-3.91, \mathrm{P}<0.001)$ greater carotid artery plaque burden compared with patients who had the lowest $\mathrm{HbA} 1 \mathrm{c} / \mathrm{HDL}-\mathrm{C}$ ratio (Q1). Moreover, the association of $\mathrm{HbA} 1 \mathrm{c} / \mathrm{HDL}-\mathrm{C}$ with atherosclerosis remained significant in a subsample of patients with and without DM.

Conclusions: As a novel compound indicator for evaluating blood glucose homeostasis and dyslipidemia, the $\mathrm{HbA} 1 \mathrm{c} / \mathrm{HDL}-\mathrm{C}$ ratio was positively correlated with carotid atherosclerosis evaluated using the mean and maximum cIMT as well as the carotid artery plaque burden.

\section{Introduction}

Atherosclerosis, a multifactorial and systemic disease process[1, 2], can lead to cardiovascular diseases (CVD) such as coronary artery disease, ischemic stroke, and peripheral artery disease. Carotid artery intima-media thickness (cIMT) and carotid artery plaque are simple, non-invasive, and clinically accessible ultrasound markers that have been widely regarded as a surrogate for atherosclerosis status and a strong predictor of future CVD [3].

The risk factors contributing to atherosclerosis are diverse, such as diabetes mellitus (DM) and dyslipidemia [4]. The relationship between DM and atherosclerosis has been widely established in clinical research and molecular research $[5,6]$. The cIMT gradually increases during the deterioration of glucose homeostasis from normal glucose tolerance to DM [7]. Glycosylated hemoglobin A1c (HbA1c) plays an important role in monitoring glucose control, even in patients without DM $[8,9]$. Many clinical studies have described the positive relationship between $\mathrm{HbA} 1 \mathrm{c}$ and $\mathrm{cIMT}$, emphasizing the recognition of atherosclerosis development at an early stage using blood glucose monitoring [10-12]. As for dyslipidemia, in the past few decades, clinical studies and meta-analyses have consistently reported that 
high-density lipoprotein cholesterol (HDL-c) levels are negatively correlated with the risk of atherosclerotic CVD $[13,14]$. The role of HDL-C in lipid metabolism (promoting reverse cholesterol transport), inflammation (tempering inflammatory mediator), and antithrombosis determines the close connection between low HDL-C and progression of atherosclerosis $[15,16]$. However, the metabolic processes of blood glucose and lipids interact with each other. A post-hoc analysis in the Stop Atherosclerosis in Native Diabetics Study (SANDS) suggested a negative correlation between $\mathrm{HbA1c}$ and achievement of blood lipid goals [17]. The presence of DM eliminates the vascular protective effect of HDL-C, and poor blood glucose control is an independent risk factor for low HDL-C in DM [18, 19]. In turn, HDL-C can reduce blood glucose in patients with DM by increasing plasma insulin [20-22].

As a single index of DM or lipids for the evaluation of atherosclerosis status may be insufficient, comprehensively considering the effects of both blood glucose control and HDL-C can avoid the inadequacy of a single index. The $\mathrm{HbA} 1 \mathrm{c} / \mathrm{HDL}-\mathrm{C}$ ratio is proposed as an easily available index related to atherosclerosis. To our knowledge, however, there is no research focusing on this comprehensive ratio and atherosclerosis. We hypothesized that the $\mathrm{HbA} 1 \mathrm{c} / \mathrm{HDL}-\mathrm{C}$ ratio is related to subclinical atherosclerosis measured using the cIMT and carotid plaque via ultrasonography.

\section{Methods}

\section{Study population}

This retrospective study included 1654 consecutive patients with multiple cardiovascular risk factors or symptoms of suspected coronary artery disease in Guangdong Provincial People's Hospital from September 2014 to September 2015. We collected information on demographic features, health behaviors, medical history (stroke, hypertension, diabetes, and chronic kidney disease), laboratory tests, and carotid ultrasound examinations from the electronic medical records. We excluded 244 patients who had used statins within the past 3 months, 84 patients who had missing data of HbA1c or HDL-C, and 22 patients without information of carotid ultrasonography examination (Fig. 1).

This study was conducted in accordance with the Declaration of Helsinki. The study was approved by the Ethics Committee of Guangdong Provincial People's Hospital, and all patients provided their verbal informed consent.

\section{Measurement}

Carotid ultrasonography examination was performed manually by experienced sonographers, who were unaware of participants' baseline characteristics and laboratory results, using a GE Vivid E95 (GE Healthcare, Milwaukee, WI, USA) and 7.5-12 MHz phased array probe. The region of interest for cIMT measurement was located at the far wall of the bilateral carotid arteries proximal to the bifurcation, with $\geq 10 \mathrm{~mm}$ of plaque-free lesions on each side. CIMT was defined as the distance between the interface of the lumen-intima and media-adventitia. The mean cIMT was taken as the average of the cIMT values of the left and right carotid arteries. Maximum cIMT was taken as the larger cIMT value between the left and 
right carotid arteries. We defined abnormal cIMT as mean cIMT or maximum cIMT values $\geq 1 \mathrm{~mm}[23$, 24]. We also assessed the carotid plaque at three different locations: the common carotid artery, carotid bifurcation, and internal carotid artery on both the left and right sides. Carotid plaque was defined as a focal structure that encroaches into the arterial lumen at least $0.5 \mathrm{~mm}$ or $50 \%$ of the surrounding cIMT value or demonstrates a thickness $>1.5 \mathrm{~mm}$ as measured from the media-adventitia interface to the intima-lumen interface [25].

Carotid plaque score was calculated as the sum of the thickest plaque values on both the left and right sides. Two sonographers measured the cIMT and carotid plaque and determined the final results together. If there was any discrepancy, a third sonographer would be consulted, and the results would be determined through discussion.

\section{Laboratory examination}

$\mathrm{HbA} 1 \mathrm{c}$ was detected using the D-10 ${ }^{\mathrm{TM}}$ Hemoglobin A1c Program (Bio-Rad) via high pressure liquid chromatography. Blood cell counts were conducted using a Sysmex-XE5000 via impedance technology. HDL-C, LDL-C, total cholesterol (TC), triglyceride (TG), apolipoprotein A (ApoA), apolipoprotein B (ApoB), fasting blood glucose, and creatinine were measured using a Beckman AU5800 spectrophotometer via colorimetry or immunoturbidimetry.

\section{Covariates}

Hypertension was diagnosed according to the European Society of Cardiology guidelines as systolic blood pressure $\geq 140 \mathrm{mmHg}$ and/or diastolic blood pressure $\geq 90 \mathrm{mmHg}$, which is equivalent to a 24-h ambulatory blood pressure monitoring average of $\geq 130 / 80 \mathrm{mmHg}$, or a home blood pressure monitoring average of $\geq 135 / 85 \mathrm{mmHg}$ for two measurements over at least 3 days [26]. DM was diagnosed based on the presence of diabetes. If the patient had a negative history of diabetes, fasting blood glucose $\geq 7.0$ $\mathrm{mmol} / \mathrm{L}(126 \mathrm{mg} / \mathrm{dL})$ and hemoglobin $\mathrm{A} 1 \mathrm{c} \geq 6.5 \%$ or positive oral glucose tolerance test (2-h plasma glucose $\geq 11.1 \mathrm{mmol} / \mathrm{L}$ [200 mg/dL]) or random plasma glucose $\geq 11.1 \mathrm{mmol} / \mathrm{L}(200 \mathrm{mg} / \mathrm{dL})$ were adopted to define DM according to the European Society of Cardiology guidelines [27]. Smoking was defined as previous smoking, and alcohol consumption was defined as previous drinking habit.

\section{Statistical analysis}

Descriptive statistics were conducted using Kruskal-Wallis $\mathrm{H}$ tests and chi-square or Fisher's exact tests for continuous and dichotomous variables, respectively. We explored the relationship between $\mathrm{HbA} 1 \mathrm{c} / \mathrm{HDL}-\mathrm{C}$ ratio and abnormal cIMT or carotid artery plaque using logistic regression. The regression results are reported according to $\mathrm{HbA} 1 \mathrm{c} / \mathrm{HDL}-\mathrm{C}$ ratio quartiles (as a categorical variable) using the lowest quartile (Q1) as reference. Odds ratios (ORs) with 95\% confidence intervals (Cls) were calculated for the associations, adjusting for age, sex, smoking, alcohol consumption, history of hypertension, LDL-C, white blood cell counts, and creatinine level. We used restricted cubic spline curves to visually assess the relationships between $\mathrm{HbA} 1 \mathrm{c} / \mathrm{HDL}-\mathrm{C}$ ratio (as a continuous variable) and abnormal clMT or carotid artery plaque. ORs and $95 \% \mathrm{Cls}$ were derived from restricted cubic spline regression, with knots placed at the 
25th, 50th, 75th, and 95th percentiles of the distribution of $\mathrm{HbA} 1 \mathrm{c} / \mathrm{HDL}-\mathrm{C}$ ratio. The reference value of the $\mathrm{HbA1} / \mathrm{HDL}-\mathrm{C}$ ratio was the median of the reference group.

The proportion of missing data in the sample for analysis was not greater than $5 \%$; missing data were interpolated using the mean value. Associations with $\mathrm{P}<0.05$ (two-sided) were considered to be statistically significant. All of the analyses were performed using Stata 15.0 (StataCorp LLC, College Station, TX, USA), R version 3.6.1 (The R Project for Statistical Computing, Vienna, Austria), and EmpowerStats (X\&Y Solutions, Inc., Boston, MA, USA).

\section{Results}

\section{Patient population}

A total of 1304 patients with complete data were included in this retrospective study (including 897 men and 407 women). The mean age (standard deviation) was 63 (11) years. The median $\mathrm{HbA} 1 \mathrm{c} / \mathrm{HDL}-\mathrm{C}$ ratio was 5.85 (interquartile range [IQR] 4.78-7.30). Baseline information of the study population is shown in Table 1. According to quartiles of the $\mathrm{HbA1}$ / $/ \mathrm{HDL}-\mathrm{C}$ ratio, patients were divided into four groups (Q1-Q4). Patients with the highest $\mathrm{HbA} 1 \mathrm{c} / \mathrm{HDL}-\mathrm{C}$ ratio (Q4) mostly comprised men and had higher values of $\mathrm{HbA1c}$, fasting blood glucose, $\mathrm{CIMT}$, and prevalence of carotid plaque, as well as lower HDL-C and ApoA. The HbA1c/HDL-C ratio was positively correlated with hypertension and white blood cells. Baseline information of the study population grouped by the presence or absence of DM is shown in Tables S1 and S2. 
Table 1

Baseline information according to quartiles of $\mathrm{HbA} 1 \mathrm{c} / \mathrm{HDL}-\mathrm{C}$ ratio

Characteristics

$\mathrm{HbA1c/HDL-C}$ ratio

$P$ value

$\begin{array}{llll}\text { Q1 (<4.78) } & \text { Q2 (4.78- } & \text { Q3 (5.85- } & \text { Q4 }>7.30 \\ & 5.85) & 7.30) & \end{array}$

Number of patients

$n=326$

$\mathrm{n}=326$

$n=326$

$n=326$

Age, years

$64.4 \pm 10.3$

$62.4 \pm 10.5$

$61.7 \pm 11.0$

$63.7 \pm 10.7$

0.007

$\mathrm{HbA} 1 \mathrm{c}, \%$

$5.7(5.4-\quad 5.9(5.6-6.2)$

6.0)

$6.1(5.7-6.6)$

$7.2(6.2-$

$<0.001$

TG, mmol/L

$1.3 \pm 0.8$

$1.4 \pm 0.7$

$1.9 \pm 1.7$

8.5)

TC, mmol/L

$4.9 \pm 1.2$

$4.6 \pm 1.2$

$4.4 \pm 1.2$

$1.9 \pm 1.4$

$<0.001$

ApoA, mmol/L†

$1.5 \pm 0.3$

$1.2 \pm 0.2$

$1.1 \pm 0.2$

$4.0 \pm 1.2$

$<0.001$

ApoB, mmol/L†

$0.8 \pm 0.3$

$0.8 \pm 0.2$

$0.8 \pm 0.2$

$1.0 \pm 0.2$

$<0.001$

LDL-C, $\mathrm{mmol} / \mathrm{L}$

$2.8 \pm 1.1$

$2.8 \pm 1.1)$

$2.6 \pm 1.1)$

$0.8 \pm 0.2$

0.067

$\mathrm{HDL}-\mathrm{C}, \mathrm{mmol} / \mathrm{L}$

$1.4 \pm 0.2$

$1.1 \pm 0.1$

$1.0 \pm 0.1$

$2.4 \pm 1.0$

$<0.001$

Creatinine, $\mathrm{mmol} / \mathrm{L} \quad 78.0 \pm 22.5$

$85.4 \pm 31.9$

$95.0 \pm 56.0$

$0.8 \pm 0.2$

$<0.001$

Mean-clMT, mm

$1.0(0.8-$

$1.0(0.8-1.1)$

1.1)

$1.0(0.8-1.1)$

$105.2 \pm 88.7$

$<0.001$

Maximum clMT, mm

$0.9(0.8-$
$1.0)^{(-1}$

$0.9(0.8-1.0)$

1.0)

Male sex

$170(52.1 \%)$

219 (67.2\%)

252 (77.3\%)

$1.1(1.0-$

1.2)

Smoking

$87(26.7 \%)$

105 (32.2\%)

115 (35.3\%)

$1.0(0.9-$

1.1)

Alcohol consumption

$18(5.5 \%)$

$24(7.4 \%)$

$25(7.7 \%)$

256 (78.5\%)

$<0.001$

Hypertension

$159(48.8 \%)$

190 (58.3\%)

202 (62.0\%)

34 (\%)

$79(24.2 \%)$

$3.1(0.0-4.4)$

$3.5(1.5-4.9)$

$2.5(0.0$
$4.3)$

Carotid artery plaque

$222(68.1 \%)$

$237(72.7 \%)$

$250(76.7 \%)$

$129(39.6 \%)$

0.005

Carotid plaque score

$23(7.1 \%)$

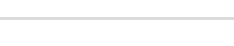

Data shown as mean \pm standard deviation or median (Q1-Q3) or $\mathrm{n}(\%)$.

tMissing data were interpolated using the mean value.

$H b A 1 c$ hemoglobin Alc, $H D L-C$ high-density lipoprotein cholesterol, $T G$ triglyceride, $T C$ total cholesterol, $A p o A$ apolipoprotein A, $A p o B$ apolipoprotein B, $L D L-C$ low-density lipoprotein cholesterol, CIMT carotid intima-media thickness, WBC white blood cells, DM diabetes mellitus 


\begin{tabular}{|c|c|c|c|c|c|}
\hline \multirow[t]{2}{*}{ Characteristics } & \multicolumn{4}{|c|}{ HbA1c/HDL-C ratio } & \multirow[t]{2}{*}{$P$ value } \\
\hline & Q1 (<4.78) & $\begin{array}{l}\text { Q2 (4.78- } \\
5.85)\end{array}$ & $\begin{array}{l}\text { Q3 (5.85- } \\
7.30)\end{array}$ & Q4 > 7.30 & \\
\hline WBC, $\times 10^{9} / \mathrm{L}$ & $7.0 \pm 2.1$ & $7.3 \pm 2.1$ & $7.7 \pm 2.3$ & $7.8 \pm 2.3$ & $<0.001$ \\
\hline $\begin{array}{l}\text { Fasting blood } \\
\text { glucose }\end{array}$ & $5.2 \pm 1.0$ & $5.4 \pm 1.2$ & $5.7 \pm 1.4$ & $7.3 \pm 2.8$ & $<0.001$ \\
\hline \multicolumn{6}{|c|}{ Data shown as mean \pm standard deviation or median (Q1-Q3) or $\mathrm{n}(\%)$. } \\
\hline \multicolumn{6}{|c|}{ tMissing data were interpolated using the mean value. } \\
\hline \multicolumn{6}{|c|}{$\begin{array}{l}\text { HbA1c hemoglobin Alc, } H D L-C \text { high-density lipoprotein cholesterol, } T G \text { triglyceride, } T C \text { total } \\
\text { cholesterol, } A p O A \text { apolipoprotein } A, A p O B \text { apolipoprotein } B, L D L-C \text { low-density lipoprotein cholesterol, } \\
c I M T \text { carotid intima-media thickness, WBC white blood cells, } D M \text { diabetes mellitus }\end{array}$} \\
\hline
\end{tabular}

\section{$\mathrm{HbA1C/HDL-C}$ ratio and cIMT or carotid artery plaque}

As shown in Fig. 2, the mean cIMT and maximum cIMT showed an upward trend with increased $\mathrm{HbA1} / \mathrm{HDL}-\mathrm{C}$ ratio $(\mathrm{P}<0.001)$. Table 2 presents results for the relationship between the $\mathrm{HbA} 1 \mathrm{c} / \mathrm{HDL}-\mathrm{C}$ ratio and abnormal cIMT or carotid artery plaque. In the unadjusted model, the OR $(95 \% \mathrm{Cl})$ for quartile 4 (Q4) of the HbA1c/HDL-C ratio was 2.55 (1.86-3.51) for abnormal mean cIMT, 3.22 (2.29-4.52) for abnormal maximum cIMT, and 2.47 (1.69-3.60) for carotid artery plaque compared with the lowest quartile. In the age- and sex-adjusted model, Q4 showed a 2.47-times (1.78-3.45) more abnormal mean cIMT, 3.20 times (2.25-4.57) more abnormal maximum cIMT, and 2.50-times (1.68-3.71) greater carotid artery plaque burden than patients with an $\mathrm{HbA} 1 \mathrm{c} / \mathrm{HDL}-\mathrm{C}$ ratio in Q1. After further adjusting for smoking, alcohol consumption, and hypertension history, the ORs (95\% Cl) of Q4 were $2.32(1.66-3.25)$ for risk of abnormal mean cIMT, 2.95 (2.05-4.21) for risk of abnormal maximum cIMT, and 2.30 (1.54-3.45) for carotid artery plaque, as compared with those of Q1. In the fully adjusted model, Q4 showed 2.88-times (2.02-4.10) abnormal mean cIMT, 3.72-times (2.55-5.44) abnormal maximum cIMT, and 2.58-times (1.70-3.91) greater carotid artery plaque burden in comparison with patients who had an $\mathrm{HbA} 1 \mathrm{c} / \mathrm{HDL}-\mathrm{C}$ ratio in Q1. In the fully adjusted model, for every 1-unit increase in the $\mathrm{HbA} 1 \mathrm{c} / \mathrm{HDL}-\mathrm{C}$ ratio, the risk was increased by $20 \%$ (OR:1.20, 95\% Cl: 1.13-1.28) for abnormal mean cIMT, by $24 \%$ (OR: 1.24, 95\% Cl: $1.16-$ 1.33) for abnormal maximum cIMT, and by $13 \%$ (OR: $1.13,95 \% \mathrm{Cl}: 1.05-1.21)$ for carotid plaque. The restrictive cubic spline curve showed that, after full adjustment for the same variables as in model 4 (Table 2), the HbA1 c/HDL-C ratio had an S-shaped relationship with abnormal average clMT (Fig. 3a), abnormal maximum clMT (Fig. 3b), and carotid plaque (Fig. 3c). 
Table 2

OR ( $95 \% \mathrm{Cls})$ of abnormal cIMT and carotid artery plaque by quartiles of $\mathrm{HbA} 1 \mathrm{c} / \mathrm{HDL}-\mathrm{C}$ ratio

$\mathrm{HbA} 1 \mathrm{c} / \mathrm{HDL}-\mathrm{C}$

Each 1-unit increase

$\begin{array}{llllll}\text { Q1 }(< & \text { Q2 (4.78- } & \text { Q3 }(5.85- & \text { Q4 }(>7.30) & \begin{array}{l}\mathrm{P} \text { for } \\ \text { trend }\end{array} & \begin{array}{l}\text { in HbA1c/HDL- } \\ \text { C ratio }\end{array} \\ \text { 4.78) } & 5.85) & 7.30) & & \end{array}$

Abnormal mean clMT ( $\geq 1 \mathrm{~mm})$

\begin{tabular}{|c|c|c|c|c|c|c|}
\hline $\begin{array}{l}\text { Median } \\
\text { HbA1c/HDL-C }\end{array}$ & 4.17 & 5.25 & 6.53 & 8.67 & - & - \\
\hline Model 1† & Ref. & $\begin{array}{l}1.10(0.81- \\
1.50)\end{array}$ & $\begin{array}{l}1.41(1.04- \\
1.92)\end{array}$ & $\begin{array}{l}2.55(1.86- \\
3.51)\end{array}$ & $<.001$ & $\begin{array}{l}1.17(1.11- \\
1.23)\end{array}$ \\
\hline Model 2† & Ref. & $\begin{array}{l}1.13(0.82- \\
1.55)\end{array}$ & $\begin{array}{l}1.45(1.05- \\
2.00)\end{array}$ & $\begin{array}{l}2.47(1.78- \\
3.45)\end{array}$ & $\begin{array}{l}< \\
0.001\end{array}$ & $\begin{array}{l}1.17(1.10- \\
1.23)\end{array}$ \\
\hline Model 3† & Ref. & $\begin{array}{l}1.10(0.79- \\
1.50)\end{array}$ & $\begin{array}{l}1.38(1.00- \\
1.91)\end{array}$ & $\begin{array}{l}2.32(1.66- \\
3.25)\end{array}$ & $<.001$ & $\begin{array}{l}1.16(1.09- \\
1.22)\end{array}$ \\
\hline Model 4† & Ref. & $\begin{array}{l}1.12(0.81- \\
1.56)\end{array}$ & $\begin{array}{l}1.54(1.10- \\
2.16)\end{array}$ & $\begin{array}{l}2.88(2.02- \\
4.10)\end{array}$ & $\begin{array}{l}< \\
0.001\end{array}$ & $\begin{array}{l}1.20(1.13- \\
1.28)\end{array}$ \\
\hline
\end{tabular}

Abnormal maximum clMT ( $\geq 1 \mathrm{~mm})$

\begin{tabular}{|c|c|c|c|c|c|c|}
\hline Model 1† & Ref. & $\begin{array}{l}1.16(0.85- \\
1.58)\end{array}$ & $\begin{array}{l}1.48(1.08- \\
2.02)\end{array}$ & $\begin{array}{l}3.22(2.29- \\
4.52)\end{array}$ & $\begin{array}{l}<.001 \\
0 .\end{array}$ & $\begin{array}{l}1.20(1.13- \\
1.28)\end{array}$ \\
\hline Model 2† & Ref. & $\begin{array}{l}1.20(0.88- \\
1.66)\end{array}$ & $\begin{array}{l}1.55(1.12- \\
2.15)\end{array}$ & $\begin{array}{l}3.20(2.25- \\
4.57)\end{array}$ & $\begin{array}{l}<.001 \\
0.01\end{array}$ & $\begin{array}{l}1.21(1.14- \\
1.28)\end{array}$ \\
\hline Model 3† & Ref. & $\begin{array}{l}1.15(0.83- \\
1.59)\end{array}$ & $\begin{array}{l}1.45(1.04- \\
2.02)\end{array}$ & $\begin{array}{l}2.95(2.05- \\
4.21)\end{array}$ & $\begin{array}{l}< \\
0.001\end{array}$ & $\begin{array}{l}1.19(1.12- \\
1.26)\end{array}$ \\
\hline Model 4† & Ref. & $\begin{array}{l}1.19(0.85- \\
1.65)\end{array}$ & $\begin{array}{l}1.63(1.16- \\
2.30)\end{array}$ & $\begin{array}{l}3.72(2.55- \\
5.44)\end{array}$ & $\begin{array}{l}< \\
0.001\end{array}$ & $\begin{array}{l}1.24(1.16- \\
1.33)\end{array}$ \\
\hline
\end{tabular}

Carotid artery plaque

$\begin{array}{lllllll}\text { Model } 1 \dagger & \text { Ref. } & 1.25(0.89- & 1.54(1.09- & 2.47(1.69- & < & 1.12(1.05- \\ & & 1.75) & 2.18) & 3.60) & 0.001 & 1.19) \\ \text { Model } 2 \dagger & \text { Ref. } & 1.34(0.94- & 1.69(1.17- & 2.50(1.68- & < & 1.12(1.05- \\ & & 1.91) & 2.45) & 3.71) & 0.001 & 1.20)\end{array}$

Data was shown as OR $(95 \% \mathrm{Cl})$ which was evaluated using logistic regression models.

†Model 1: unadjusted; Model 2: adjusted for age and sex; Model 3: further adjusted for smoking, alcohol consumption, and history of hypertension; Model 4: further adjusted for LDL-C, white blood cell count, and creatinine level.

$H b A 1 c$ hemoglobin Alc, $H D L-C$ high-density lipoprotein cholesterol, $c I M T$ carotid intima-media thickness, Ref reference, $L D L-C$ low-density lipoprotein cholesterol, $C /$ confidence interval 


\begin{tabular}{|c|c|c|c|c|c|c|}
\hline & \multicolumn{5}{|c|}{ HbA1c/HDL-C } & \multirow{2}{*}{$\begin{array}{l}\text { Each 1-unit } \\
\text { increase } \\
\text { in } \mathrm{HbA1c/HDL-} \\
\mathrm{C} \text { ratio }\end{array}$} \\
\hline & $\begin{array}{l}\text { Q1 ( } \\
4.78)\end{array}$ & $\begin{array}{l}\text { Q2 (4.78- } \\
5.85)\end{array}$ & $\begin{array}{l}\text { Q3 (5.85- } \\
7.30)\end{array}$ & Q4 (>7.30) & $\begin{array}{l}\text { P for } \\
\text { trend }\end{array}$ & \\
\hline Model $3+$ & Ref. & $\begin{array}{l}1.27(0.89- \\
1.82)\end{array}$ & $\begin{array}{l}1.58(1.09- \\
2.30)\end{array}$ & $\begin{array}{l}2.30(1.54- \\
3.45)\end{array}$ & $\begin{array}{l}<.001 \\
0 .\end{array}$ & $\begin{array}{l}1.11(1.03- \\
1.18)\end{array}$ \\
\hline Model $4 \dagger$ & Ref. & $\begin{array}{l}1.29(0.90- \\
1.86)\end{array}$ & $\begin{array}{l}1.68(1.14- \\
2.45)\end{array}$ & $\begin{array}{l}2.58(1.70- \\
3.91)\end{array}$ & $\begin{array}{l}< \\
0.001\end{array}$ & $\begin{array}{l}1.13(1.05- \\
1.21)\end{array}$ \\
\hline \multicolumn{7}{|c|}{ Data was shown as OR $(95 \% \mathrm{Cl})$ which was evaluated using logistic regression models. } \\
\hline \multicolumn{7}{|c|}{$\begin{array}{l}\text { †Model 1: unadjusted; Model 2: adjusted for age and sex; Model 3: further adjusted for smoking, } \\
\text { alcohol consumption, and history of hypertension; Model 4: further adjusted for LDL-C, white blood } \\
\text { cell count, and creatinine level. }\end{array}$} \\
\hline \multicolumn{7}{|c|}{$\begin{array}{l}\text { HbA1c hemoglobin Alc, } H D L-C \text { high-density lipoprotein cholesterol, } c I M T \text { carotid intima-media } \\
\text { thickness, Ref reference, } L D L-C \text { low-density lipoprotein cholesterol, } C / \text { confidence interval }\end{array}$} \\
\hline
\end{tabular}

In patients with $\mathrm{DM}$, after multivariable adjustment for the risk factors included in model 4 , the $\mathrm{HbA1c/HDL-C}$ ratio was significantly correlated with abnormal mean cIMT (OR: 2.87, 95\% Cl: 1.55-5.32) and abnormal maximum cIMT (OR: 4.00,95\% Cl: 2.00-8.02), but the relationship with carotid artery plaque did not reach a significant level (OR: 1.36, 95\% Cl: 0.68-2.71) (Table 3). Restricted cubic splines showed a linear relationship between HbA1c/HDL-C ratio and abnormal cIMT (Fig. 3d, 3e), but the $\mathrm{HbA} 1 \mathrm{c} / \mathrm{HDL}-\mathrm{C}$ ratio and carotid artery plaque had an inverted U-shaped relationship (Fig. $3 \mathrm{f}$ ). In patients without DM, after multivariable adjustment, the $\mathrm{HbA} 1 \mathrm{c} / \mathrm{HDL}-\mathrm{C}$ ratio was significantly correlated with abnormal mean cIMT (OR: 2.02, 95\% Cl: 1.30-3.15), abnormal maximum cIMT (OR: 1.92, 95\% Cl: 1.232.99), and carotid artery plaque (OR: $2.31,95 \% \mathrm{Cl}$ : 1.39-3.86) (Table 3 ). The restricted cubic spline curve showed a J-shaped relationship between $\mathrm{HbA} 1 \mathrm{C} / \mathrm{HDL}-\mathrm{C}$ ratio and abnormal cIMT or carotid plaque (Fig. 3g-3i). 
Table 3

The association between $\mathrm{HbA} 1 \mathrm{c} / \mathrm{HDL}-\mathrm{C}$ ratio and carotid atherosclerosis in patients with and without DM With DM $(\mathrm{N}=450)$

\begin{tabular}{|c|c|c|c|c|c|c|}
\hline & \multicolumn{5}{|c|}{$\mathrm{HbA} 1 \mathrm{c} / \mathrm{HDL}-\mathrm{C}$} & \multirow{2}{*}{$\begin{array}{l}\text { Each 1-unit } \\
\text { increase } \\
\text { in } \\
\mathrm{HbA} 1 \mathrm{c} / \mathrm{HDL}-\mathrm{C}\end{array}$} \\
\hline & $\begin{array}{l}\text { Q1 (< } \\
5.98)\end{array}$ & $\begin{array}{l}\text { Q2 (5.98- } \\
7.38)\end{array}$ & $\begin{array}{l}\text { Q3 (7.38- } \\
9.07)\end{array}$ & Q4 (>9.07) & $\begin{array}{l}P \text { for } \\
\text { trend }\end{array}$ & \\
\hline $\begin{array}{l}\text { Median } \\
\text { HbA1c/HDL-C }\end{array}$ & 5.18 & 6.77 & 8.23 & 10.73 & - & - \\
\hline \multicolumn{7}{|c|}{ Abnormal mean clMT ( $\geq 1 \mathrm{~mm})$} \\
\hline Model 1† & Ref. & $\begin{array}{l}1.63(0.96- \\
2.79)\end{array}$ & $\begin{array}{l}1.70(1.00- \\
2.90)\end{array}$ & $\begin{array}{l}2.23(1.29- \\
3.86)\end{array}$ & 0.005 & $\begin{array}{l}1.13(1.04- \\
1.22)\end{array}$ \\
\hline Model 2† & Ref. & $\begin{array}{l}1.56(0.90- \\
2.70)\end{array}$ & $\begin{array}{l}1.68(0.97- \\
2.90)\end{array}$ & $\begin{array}{l}2.29(1.28- \\
4.08)\end{array}$ & 0.006 & $\begin{array}{l}1.13(1.04- \\
1.23)\end{array}$ \\
\hline Model 3† & Ref. & $\begin{array}{l}1.53(0.88- \\
2.67)\end{array}$ & $\begin{array}{l}1.68(0.97- \\
2.92)\end{array}$ & $\begin{array}{l}2.23(1.25- \\
4.01)\end{array}$ & 0.008 & $\begin{array}{l}1.16(1.09- \\
1.22)\end{array}$ \\
\hline Model 4† & Ref. & $\begin{array}{l}1.65(0.94- \\
2.91)\end{array}$ & $\begin{array}{l}1.85(1.05- \\
3.26)\end{array}$ & $\begin{array}{l}2.87(1.55- \\
5.32)\end{array}$ & 0.001 & $\begin{array}{l}1.13(1.04- \\
1.23)\end{array}$ \\
\hline \multicolumn{7}{|c|}{ Abnormal maximum clMT ( $\geq 1 \mathrm{~mm}$ ) } \\
\hline Model 1† & Ref. & $\begin{array}{l}1.79(1.02- \\
3.15)\end{array}$ & $\begin{array}{l}2.17(1.21- \\
3.86)\end{array}$ & $\begin{array}{l}2.80(1.53- \\
5.15)\end{array}$ & 0.001 & $\begin{array}{l}1.17(1.06- \\
1.28)\end{array}$ \\
\hline Model 2† & Ref. & $\begin{array}{l}1.72(0.97- \\
3.08)\end{array}$ & $\begin{array}{l}2.17(1.20- \\
3.92)\end{array}$ & $\begin{array}{l}2.94(1.55- \\
5.57)\end{array}$ & 0.001 & $\begin{array}{l}1.17(1.07- \\
1.29)\end{array}$ \\
\hline Model 3† & Ref. & $\begin{array}{l}1.68(0.93- \\
3.02)\end{array}$ & $\begin{array}{l}2.16(1.19- \\
3.94)\end{array}$ & $\begin{array}{l}2.83(1.48- \\
5.41)\end{array}$ & 0.001 & $\begin{array}{l}1.17(1.06- \\
1.29)\end{array}$ \\
\hline Model 4† & Ref. & $\begin{array}{l}1.82(0.99- \\
3.34)\end{array}$ & $\begin{array}{l}2.44(1.30- \\
4.56)\end{array}$ & $\begin{array}{l}4.00(2.00- \\
8.02)\end{array}$ & $<0.001$ & $\begin{array}{l}1.22(1.10- \\
1.35)\end{array}$ \\
\hline \multicolumn{7}{|c|}{ Carotid artery plaque } \\
\hline Model 1† & Ref. & $\begin{array}{l}1.38(0.73- \\
2.59)\end{array}$ & $\begin{array}{l}2.02(1.02- \\
3.99)\end{array}$ & $\begin{array}{l}1.22(0.66- \\
2.28)\end{array}$ & 0.342 & $\begin{array}{l}1.03(0.94- \\
1.12)\end{array}$ \\
\hline
\end{tabular}

Data was shown as OR $(95 \% \mathrm{Cl})$ which was evaluated using logistic regression models.

tModel 1: unadjusted; Model 2: adjusted for age and sex; Model 3: further adjusted for smoking, alcohol consumption, and history of hypertension; Model 4: further adjusted for LDL-C, white blood cell count, and creatinine level.

$H b A 1 c$ hemoglobin Alc, $H D L-C$ high-density lipoprotein cholesterol, $c I M T$ carotid intima-media thickness, $R$ ef reference, $D M$ diabetes mellitus, $L D L-C$ low-density lipoprotein cholesterol, $C l$ confidence interval 
With DM $(\mathrm{N}=450)$

\begin{tabular}{|c|c|c|c|c|c|c|}
\hline Model $2 \dagger$ & Ref. & $\begin{array}{l}1.38(0.72- \\
2.65)\end{array}$ & $\begin{array}{l}2.11(1.05- \\
4.22)\end{array}$ & $\begin{array}{l}1.37(0.71- \\
2.64)\end{array}$ & 0.208 & $\begin{array}{l}1.04(0.95- \\
1.14)\end{array}$ \\
\hline Model 3† & Ref. & $\begin{array}{l}1.35(0.68- \\
2.60)\end{array}$ & $\begin{array}{l}2.09(1.04- \\
4.22)\end{array}$ & $\begin{array}{l}1.30(0.67- \\
2.54)\end{array}$ & 0.259 & $\begin{array}{l}1.04(0.94- \\
1.14)\end{array}$ \\
\hline Model 4† & Ref. & $\begin{array}{l}1.36(0.70- \\
2.63)\end{array}$ & $\begin{array}{l}2.10(1.04- \\
4.27)\end{array}$ & $\begin{array}{l}1.36(0.68- \\
2.71)\end{array}$ & 0.222 & $\begin{array}{l}1.04(0.95- \\
1.15)\end{array}$ \\
\hline
\end{tabular}

Without DM $(\mathrm{N}=854)$

HbA1c/HDL-C

Q1 $<\quad$ Q2 (4.41-

4.41)

3.96

Median

HbA1c/HDL-C
5.23)

4.88

5.74

Q3 (5.23-

6.38)

Q4 (>6.38)

P for

trend
Each 1-unit increase

in

$\mathrm{HbA1c/HDL-C}$

Abnormal mean clMT ( $\geq 1 \mathrm{~mm}$ )

\begin{tabular}{|c|c|c|c|c|c|c|}
\hline Model 1† & Ref. & $\begin{array}{l}1.27(0.87- \\
1.86)\end{array}$ & $\begin{array}{l}1.16(0.79- \\
1.69)\end{array}$ & $\begin{array}{l}1.67(1.14- \\
2.44)\end{array}$ & 0.020 & $\begin{array}{l}1.11(1.02- \\
1.22)\end{array}$ \\
\hline Model 2† & Ref. & $\begin{array}{l}1.24(0.83- \\
1.85)\end{array}$ & $\begin{array}{l}1.25(0.84- \\
1.87)\end{array}$ & $\begin{array}{l}1.61(1.07- \\
2.44)\end{array}$ & 0.032 & $\begin{array}{l}1.10(1.00- \\
1.22)\end{array}$ \\
\hline Model 3† & Ref. & $\begin{array}{l}1.24(0.83- \\
1.85)\end{array}$ & $\begin{array}{l}1.22(0.81- \\
1.82)\end{array}$ & $\begin{array}{l}1.56(1.03- \\
2.36)\end{array}$ & 0.052 & $\begin{array}{l}1.09(0.99- \\
1.20)\end{array}$ \\
\hline Model 4† & Ref. & $\begin{array}{l}2.02(0.81- \\
1.56)\end{array}$ & $\begin{array}{l}1.54(1.10- \\
2.16)\end{array}$ & $\begin{array}{l}2.02(1.30- \\
3.15)\end{array}$ & 0.002 & $\begin{array}{l}1.16(1.05- \\
1.29)\end{array}$ \\
\hline
\end{tabular}

Abnormal maximum clMT ( $\geq 1 \mathrm{~mm})$

Model 1†

Ref.

$1.11(0.76-$

$1.11(0.76-$

$1.63(1.10-\quad 0.021$

$1.13(1.03-$

1.62)

1.62)

2.40)

1.24)

Model 2†

Ref.

1.09 (0.73-

1.62)

$1.21(0.81-$

$1.60(1.06-\quad 0.025$

$1.13(1.02-$

1.81)

2.44)

1.25)

Model 3†

Ref.

$1.08(0.72-$

1.15 (0.77-

1.73)

$1.52(1.00-$

0.051

$1.11(1.00-$

1.23)

Data was shown as OR $(95 \% \mathrm{Cl})$ which was evaluated using logistic regression models.

tModel 1: unadjusted; Model 2: adjusted for age and sex; Model 3: further adjusted for smoking, alcohol consumption, and history of hypertension; Model 4: further adjusted for LDL-C, white blood cell count, and creatinine level.

$H b A 1 c$ hemoglobin Alc, $H D L-C$ high-density lipoprotein cholesterol, $c I M T$ carotid intima-media thickness, Ref reference, $D M$ diabetes mellitus, $L D L-C$ low-density lipoprotein cholesterol, $C l$ confidence interval 


\begin{tabular}{|c|c|c|c|c|c|c|}
\hline \multicolumn{7}{|c|}{ With DM $(N=450)$} \\
\hline Model 4† & Ref. & $\begin{array}{l}1.09(0.73- \\
1.65)\end{array}$ & $\begin{array}{l}1.25(0.83- \\
1.90)\end{array}$ & $\begin{array}{l}1.92(1.23- \\
2.99)\end{array}$ & 0.004 & $\begin{array}{l}1.18(1.06- \\
1.31)\end{array}$ \\
\hline \multicolumn{7}{|c|}{ Carotid artery plaque } \\
\hline Model $1 \dagger$ & Ref. & $\begin{array}{l}1.42(0.93- \\
2.16)\end{array}$ & $\begin{array}{l}1.05(0.70- \\
1.58)\end{array}$ & $\begin{array}{l}2.01(1.29- \\
3.14)\end{array}$ & 0.015 & $\begin{array}{l}1.20(1.07- \\
1.34)\end{array}$ \\
\hline Model $2 \dagger$ & Ref. & $\begin{array}{l}1.46(0.94- \\
2.28)\end{array}$ & $\begin{array}{l}1.20(0.78- \\
1.86)\end{array}$ & $\begin{array}{l}2.10(1.29- \\
3.40)\end{array}$ & 0.011 & $\begin{array}{l}1.22(1.08- \\
1.38)\end{array}$ \\
\hline Model 3† & Ref. & $\begin{array}{l}1.41(0.89- \\
2.21)\end{array}$ & $\begin{array}{l}1.12(0.72- \\
1.74)\end{array}$ & $\begin{array}{l}1.98(1.21- \\
3.25)\end{array}$ & 0.027 & $\begin{array}{l}1.20(1.06- \\
1.36)\end{array}$ \\
\hline Model 4† & Ref. & $\begin{array}{l}1.43(0.90- \\
2.27)\end{array}$ & $\begin{array}{l}1.17(0.74- \\
1.83)\end{array}$ & $\begin{array}{l}2.31(1.39- \\
3.86)\end{array}$ & 0.007 & $\begin{array}{l}1.25(1.10- \\
1.43)\end{array}$ \\
\hline \multicolumn{7}{|c|}{ Data was shown as OR $(95 \% \mathrm{Cl})$ which was evaluated using logistic regression models. } \\
\hline \multicolumn{7}{|c|}{$\begin{array}{l}\text { †Model 1: unadjusted; Model 2: adjusted for age and sex; Model 3: further adjusted for smoking, } \\
\text { alcohol consumption, and history of hypertension; Model 4: further adjusted for LDL-C, white blood } \\
\text { cell count, and creatinine level. }\end{array}$} \\
\hline \multicolumn{7}{|c|}{$\begin{array}{l}\text { HbA1c hemoglobin Alc, } H D L-C \text { high-density lipoprotein cholesterol, } c I M T \text { carotid intima-media } \\
\text { thickness, Ref reference, } D M \text { diabetes mellitus, } L D L-C \text { low-density lipoprotein cholesterol, } C l \\
\text { confidence interval }\end{array}$} \\
\hline
\end{tabular}

\section{Subgroup analysis}

Subgroup analysis showed that the above covariates (age, sex, smoking, alcohol consumption, and hypertension history) did not change the relationship between the HbA1c/HDL-C ratio and abnormal cIMT or carotid plaque ( $P$ for interaction $>0.05$; Table S3).

\section{Discussion}

In the present study, we proposed a composite indicator of blood glucose homeostasis and dyslipidemia, the $\mathrm{HbA} 1 \mathrm{c} / \mathrm{HDL}-\mathrm{C}$ ratio, and demonstrated the relationship between this ratio and subclinical carotid atherosclerosis. We observed a positive correlation between $\mathrm{HbA1c/HDL-C}$ levels and abnormal mean/maximum cIMT as well as carotid artery plaque burden in patients with a high risk of CVD, independent of traditional clinical risk factors. More importantly, the relationship between $\mathrm{HbA} 1 \mathrm{c} / \mathrm{HDL}-\mathrm{C}$ and abnormal cIMT remained in a subsample of patients with and without DM. HbA1c/HDL-C may be an important biomarker for clinical evaluation of atherosclerosis progression.

Atherosclerosis is a slow, gradually progressing disease that begins in childhood, but there are potential risk factors that can accelerate its development such as poor glycemic control and low HDL-C [14, 28]. Patients with DM are four times more likely to develop CVD than individuals who do not have DM, and DM is also related to poor clinical prognosis of CVD events [29]. Hyperglycemia is the predominant feature of DM. Many studies have shown that the extent of glycemic control, sensitively reflected by 
$\mathrm{HbA1c}$, is related to CVD complications in patients with DM [30, 31]. A cross-sectional study involving 541 Chinese people suggested that $\mathrm{HbA} 1 \mathrm{c}$ levels are significantly associated with cIMT and the presence of carotid artery plaque [10]. In addition, a multi-detector coronary computed tomography data analysis based on an observational cohort study on the clinical outcomes in asymptomatic patients with type 2 DM showed that long-term stable HbA1c levels may be of great importance in preventing subclinical coronary atherosclerosis in patients with DM [32]. Moreover, a clinical trial, the UK Prospective Diabetes Study (UKPDS), indicated that well-controlled blood glucose and $\mathrm{HbA} 1 \mathrm{c}$ could reduce the progression of CVD in DM [33]. Low HDL-C is an important manifestations of dyslipidemia. Clinical studies have consistently shown that low HDL-C is an independent risk factor for atherosclerotic CVD [14, 34]. A metaanalysis involving 21,000 people concluded that elevated CIMT is associated with low HDL-C levels, independent of LDL-C levels. Even in patients who are treated aggressively with statins to decrease LDLC, HDL-C is still a major predictor of CVD development [35]. In addition, a study synthesizing the data of three cohorts, namely, the European Prospective Investigation into Cancer (EPIC) Norfolk Study, the Atherosclerosis Risk In Communities Study (ARIC), and the Women's Health Study, verified that ApoA levels cannot provide additional predictive information over HDL-C for CVD [36]. Therefore, levels of $\mathrm{HbA1C}$ and HDL-C are useful indicators that reflect DM and dyslipidemia, respectively, which are closely related to the progression of atherosclerosis. Our study puts forth a new marker, the HbA1c/HDL-C ratio, which was found to be significantly associated with abnormal cIMT and carotid artery plaque after adjustment for other relevant clinical covariates. The present findings were consistent with those of previous studies on $\mathrm{HbA} 1 \mathrm{c}$ and $\mathrm{HDL}-\mathrm{C}$ [32-35]. Furthermore, the $\mathrm{HbA} 1 \mathrm{c} / \mathrm{HDL}-\mathrm{C}$ ratio takes into account the relative state of blood glucose control and lipid levels. In recent years, studies have found that blood glucose homeostasis has an impact on blood lipid metabolism and vice versa. Kinochi et al. proposed that the existence of DM eliminates the vascular protective effects of both HDL-C on endothelial function and the progression of atherosclerosis [19]. In a post-hoc analysis of the SANDS study, it was found that levels of $\mathrm{HbA} 1 \mathrm{c}$ were negatively correlated with the ability to achieve blood lipid targets with lipidlowering treatment among patients with DM [17]. In contrast, HDL-C has potent antidiabetic properties via increasing insulin sensitivity and $\beta$-cell function, reflecting its role in regulating blood glucose homeostasis [20-22]. In this study, the HbA1 C/HDL-C ratio marker could comprehensively reflect blood glucose homeostasis and dyslipidemia, which can be used to monitor progression of atherosclerosis.

The deterioration of blood glucose homeostasis and dyslipidemia can lead to atherosclerosis by impairing endothelial function, increasing inflammation, reducing cholesterol outflow, and promoting platelet aggregation [6,37]. Metabolic abnormalities of DM, such as hyperglycemia, increased free fatty acids, and insulin resistance, directly result in vascular dysfunction. The molecular mechanisms include the reduction of nitric oxide bioavailability, the increase of oxidative stress, and the interference with intracellular signal transduction [38]. A reduction in HDL-C activates local inflammation and endothelial thrombosis, increases endothelial cell apoptosis, and slows down vascular repair [37]. With the double attack of increasing $\mathrm{HbA} 1 \mathrm{c}$ and decreasing $\mathrm{HDL}-\mathrm{C}$, the progression of atherosclerosis will rapidly accelerate. From this point of view, the $\mathrm{HbA} 1 \mathrm{c} / \mathrm{HDL}-\mathrm{C}$ ratio could reflect the relative status of blood glucose and serum lipids as well as the extent of damage to the arterial endothelium, which in turn can 
explain the rationality of the $\mathrm{HbA} 1 \mathrm{c} / \mathrm{HDL}-\mathrm{C}$ ratio as a marker of atherosclerosis progression through the above pathophysiological mechanism.

Another finding of our study was that in a subsample with and without DM, the HbA1c/HDL-C ratio was still associated with abnormal cIMT. Blood glucose homeostasis as reflected by HbA1c is not only applicable in the population with DM but also useful in those without diabetes. A recent cohort study showed that the use of $\mathrm{HbA} 1 \mathrm{c}$ can better identify subclinical atherosclerosis in individuals without $\mathrm{DM}$, on the top of traditional CVD prediction models [9]. Bobbert et al. found that in patients without DM, $\mathrm{HbA1c}$ had a stronger correlation with cIMT than fasting or 2-h glucose levels [39]. Because blood glucose homeostasis in people without DM will also affect subclinical atherosclerosis, HbA1c levels also require attention. Therefore, the application of the proposed new marker, the $\mathrm{HbA} 1 \mathrm{c} / \mathrm{HDL}-\mathrm{C}$ ratio, for individuals without DM may have an important role in primary prevention among this population.

The present research has several limitations. First, this was a single-center study; our findings should be extrapolated carefully to other populations with different demographic characteristics. Second, the crosssectional study design prevented us prospectively identifying a causal relationship between the long-term $\mathrm{HbA} 1 \mathrm{c} / \mathrm{HDL}-\mathrm{C}$ ratio and development of atherosclerosis. Third, in patients with $\mathrm{DM}$, the dose-response relationship between the $\mathrm{HbA} 1 \mathrm{c} / \mathrm{HDL}-\mathrm{C}$ ratio and carotid plaque score showed that with increased $\mathrm{HbA1c/HDL-C}$ ratio, total carotid plaque thickness was increased. Although there was no significant correlation between the $\mathrm{HbA} 1 \mathrm{c} / \mathrm{HDL}-\mathrm{C}$ ratio and presence of carotid plaque when the $\mathrm{HbA} 1 \mathrm{c} / \mathrm{HDL}-\mathrm{C}$ ratio was at high level, these still tended to be positively correlated. The reason may be an insufficient sample size in both the DM subsample and quartile groups.

\section{Conclusion}

As a new indicator for evaluating blood glucose homeostasis and dyslipidemia, elevated $\mathrm{HbA} 1 \mathrm{c} / \mathrm{HDL}-\mathrm{C}$ ratio was found to be associated with the mean and the maximum cIMT, as well as the occurrence of carotid artery plaque, independent of conventional risk factors. These associations remained in patients with and without DM. Our study findings suggest that the $\mathrm{HbA1c/HDL-C}$ ratio may be useful for monitoring high-risk patients in the primary prevention of atherosclerosis.

\section{Abbreviations}

DM: Diabetes mellitus; HbA1c: Glycosylated hemoglobin A1c; HDL-C: High-density lipoprotein cholesterol; LDL-C: Low-density lipoprotein cholesterol; cIMT: Carotid artery intima-media thickness; CVD: Cardiovascular diseases; TC: Total cholesterol; TG: Triglyceride; ApoA: Apolipoprotein-A; ApoB: Apolipoprotein-B; OR: Odds ratio; Cl: Confidence interval; IQR: Interquartile range; Q: Quantile.

\section{Declarations}

\section{Ethics approval and consent to participate}


This study was approved by the Ethics Committee of Guangdong Provincial People's Hospital. All patients provided verbal informed consent. This study was conducted in accordance with the Declaration of Helsinki.

\section{Consent for publication}

Not applicable.

\section{Availability of data and materials}

The data analyzed in this study can be obtained from the corresponding author with a reasonable request.

\section{Competing interests}

The authors declare no conflicts of interest related to this article.

\section{Funding}

Our research was supported by the National Key Research and Development Program of China (No. 2016YFC1301202).

\section{Authors' contributions}

$\mathrm{XMH}$, the first author, contributed to the manuscript preparation; WL, CYW and HTZ contributed to the data collection and collation. XMH and HYL contributed to the data analysis. GL provided critical revisions of the manuscript. YLZ and HJD contributed to the research ideas and approved the final version of the manuscript for submission. All authors read and approved the final manuscript.

\section{Acknowledgments}

We thank LetPub (www.letpub.com) for its linguistic assistance during the preparation of this manuscript.

\section{Author's information}

${ }^{1}$ The Second School of Clinical Medicine, Southern Medical University, Guangzhou 510515, Guangdong, China;

${ }^{2}$ Department of Cardiology, Guangdong Cardiovascular Institute, Guangdong Provincial Key Laboratory of Coronary Heart Disease Prevention, Guangdong Provincial People's Hospital, Guangdong Academy of Medical Sciences, Guangzhou 510080, Guangdong, China;

${ }^{3}$ Department of Cardiology, Guangdong Provincial People's Hospital 
Zhuhai Hospital (Zhuhai Golden Bay Center Hospital), Zhuhai 519040,

Guangdong, China.

\section{References}

1. Mach F, Baigent C, Catapano AL, Koskinas KC, Casula M, Badimon L, Chapman MJ, De Backer GG, Delgado V, Ference BA, et al. 2019 ESC/EAS Guidelines for the management of dyslipidaemias: lipid modification to reduce cardiovascular risk. Eur Heart J. 2020;41(1):111-88.

2. Song P, Fang Z, Wang H, Cai Y, Rahimi K, Zhu Y, Fowkes FGR, Fowkes FJI, Rudan I. Global and regional prevalence, burden, and risk factors for carotid atherosclerosis: a systematic review, metaanalysis, and modelling study. Lancet Glob Health. 2020;8(5):e721-9.

3. Sun P, Liu L, Liu C, Zhang Y, Yang Y, Qin X, Li J, Cao J, Zhang Y, Zhou Z, et al. Carotid Intima-Media Thickness and the Risk of First Stroke in Patients With Hypertension. Stroke. 2020;51(2):379-86.

4. Pacilli A, De Cosmo S, Trischitta V, Bacci S. Role of relationship between HbA1c, fibrinogen and HDLcholesterol on cardiovascular disease in patients with type 2 diabetes mellitus. Atherosclerosis. 2013;228(1):247-8.

5. Nezu T, Hosomi N, Aoki S, Matsumoto M. Carotid Intima-Media Thickness for Atherosclerosis. J Atheroscler Thromb. 2016;23(1):18-31.

6. La Sala L, Prattichizzo F, Ceriello A. The link between diabetes and atherosclerosis. Eur J Prev Cardiol. 2019;26(2_suppl):15-24.

7. Fiorentino TV, Marini MA, Succurro E, Andreozzi F, Sesti G. Relationships of surrogate indexes of insulin resistance with insulin sensitivity assessed by euglycemic hyperinsulinemic clamp and subclinical vascular damage. BMJ Open Diabetes Res Care. 2019;7(1):e000911.

8. Selvin E, Crainiceanu CM, Brancati FL, Coresh J. Short-term variability in measures of glycemia and implications for the classifification of diabetes. Arch Intern Med. 2007;167:1545-51.

9. Rossello X, Raposeiras-Roubin S, Oliva B, Sánchez-Cabo F, García-Ruíz JM, Caimari F, Mendiguren JM, Lara-Pezzi E, Bueno H, Fernández-Friera L, et al. Glycated Hemoglobin and Subclinical Atherosclerosis in People Without Diabetes. J Am Coll Cardiol. 2021;77(22):2777-91.

10. Hung CS, Lee PC, Li HY, Ma WY, Lin MS, Wei JN, Shih SR, Hua CH, Chuang LM, Chen MF. Haemoglobin A1 $\mathrm{c}$ is associated with carotid intima-media thickness in a Chinese population. Clin Endocrinol (Oxf). 2011;75(6):780-5.

11. Shah AS, Dolan LM, Kimball TR, Gao Z, Khoury PR, Daniels SR, Urbina EM. Influence of duration of diabetes, glycemic control, and traditional cardiovascular risk factors on early atherosclerotic vascular changes in adolescents and young adults with type 2 diabetes mellitus. J Clin Endocrinol Metab. 2009;94(10):3740-5.

12. Larsen JR, Brekke M, Bergengen L, Sandvik L, Arnesen H, Hanssen KF, Dahl-Jorgensen K. Mean $\mathrm{HbA} 1 \mathrm{c}$ over 18 years predicts carotid intima media thickness in women with type 1 diabetes. Diabetologia. 2005;48(4):776-9. 
13. Prospective Studies Collaboration. Lewington S, Whitlock G, Clarke R, Sherliker P, Emberson J, Halsey J, Qizilbash N, Peto R, Collins R. Blood cholesterol and vascular mortality by age, sex, and blood pressure: a meta-analysis of individual data from 61 prospective studies with 55,000 vascular deaths. Lancet. 2007;370(9602):1829-39.

14. Emerging Risk Factors Collaboration. Di Angelantonio E, Sarwar N, Perry P, Kaptoge S, Ray KK, Thompson A, Wood AM, Lewington S, Sattar N, et al. Major lipids, apolipoproteins, and risk of vascular disease. JAMA. 2009;302(18):1993-2000.

15. Parhofer KG. Increasing HDL-cholesterol and prevention of atherosclerosis: A critical perspective. Atheroscler Suppl. 2015;18:109-11.

16. Mineo C, Shaul PW. Novel biological functions of high-density lipoprotein cholesterol. Circ Res. 2012;111(8):1079-90.

17. Mete M, Wilson C, Lee ET, Silverman A, Russell M, Stylianou M, Umans JG, Wang W, Howard WJ, Ratner RE, et al. Relationship of glycemia control to lipid and blood pressure lowering and atherosclerosis: the SANDS experience. J Diabetes Complications. 2011;25(6):362-7.

18. Gatti A, Maranghi M, Bacci S, Carallo C, Gnasso A, Mandosi E, Fallarino M, Morano S, Trischitta V, Filetti S. Poor glycemic control is an independent risk factor for low HDL cholesterol in patients with type 2 diabetes. Diabetes Care. 2009;32(8):1550-2.

19. Kinouchi M, Aihara K, Fujinaka Y, Yoshida S, Ooguro Y, Kurahashi K, Kondo T, Aki N, Kuroda A, Endo I, et al. Diabetic conditions differentially affect the endothelial function, arterial stiffness and carotid atherosclerosis. J Atheroscler Thromb. 2014;21(5):486-500.

20. Cochran BJ, Ong KL, Manandhar B, Rye KA. High Density Lipoproteins and Diabetes. Cells. 2021;10(4):850.

21. Drew BG, Duffy SJ, Formosa MF, Natoli AK, Henstridge DC, Penfold SA, Thomas WG, Mukhamedova $\mathrm{N}$, de Courten B, Forbes JM, et al. High-density lipoprotein modulates glucose metabolism in patients with type 2 diabetes mellitus. Circulation. 2009;119(15):2103-11.

22. Manandhar B, Cochran BJ, Rye KA. Role of High-Density Lipoproteins in Cholesterol Homeostasis and Glycemic Control. J Am Heart Assoc. 2020;9(1):e013531.

23. Chambless LE, Heiss G, Folsom AR, Rosamond W, Szklo M, Sharrett AR, Clegg LX. Association of coronary heart disease incidence with carotid arterial wall thickness and major risk factors: the atherosclerosis risk in communities (aric) study, 1987-1993. Am J Epidemiol. 1997;146:483-94.

24. Naqvi TZ, Lee MS. Carotid intima-media thickness and plaque in cardiovascular risk assessment. JACC Cardiovasc Imaging. 2014;7:1025-38.

25. Touboul PJ, Hennerici MG, Meairs S, Adams H, Amarenco P, Bornstein N, Csiba L, Desvarieux M, Ebrahim S, Hernandez Hernandez R, et al Mannheim carotid intima-media thickness and plaque consensus (2004-2006-2011). An update on behalf of the advisory board of the 3rd, 4th and 5th watching the risk symposia, at the 13th, 15th and 20th European Stroke Conferences, Mannheim, Germany, 2004, Brussels, Belgium, 2006, and Hamburg G. 2011. Cerebrovasc Dis. 2012;34(4):290296. 
26. Williams B, Mancia G, Spiering W, Agabiti Rosei E, Azizi M, Burnier M, Clement DL, Coca A, de Simone G, Dominiczak A, et al. 2018 ESC/ESH Guidelines for the management of arterial hypertension. Eur Heart J. 2018;39(33):3021-104.

27. Cosentino F, Grant PJ, Aboyans V, Bailey CJ, Ceriello A, Delgado V, Federici M, Filippatos G, Grobbee DE, Hansen TB, et al. 2019 ESC Guidelines on diabetes, pre-diabetes, and cardiovascular diseases developed in collaboration with the EASD. Eur Heart J. 2020;41(2):255-323.

28. Wagenknecht LE, Zaccaro D, Espeland MA, Karter AJ, O'Leary DH, Haffner SM. Diabetes and progression of carotid atherosclerosis: the insulin resistance atherosclerosis study. Arterioscler Thromb Vasc Biol. 2003;23(6):1035-41.

29. Beckman JA, Creager MA, Libby P. Diabetes and atherosclerosis: epidemiology, pathophysiology, and management. JAMA. 2002;287(19):2570-81.

30. Hayward RA, Reaven PD, Wiitala WL, Bahn GD, Reda DJ, Ge L, Investigators VADT, et al. Follow-up of glycemic control and cardiovascular outcomes in type 2 diabetes. N Engl J Med. 2015;372:2197206.

31. Reaven PD, Emanuele NV, Wiitala WL, Bahn GD, Reda DJ, McCarren M, Duckworth WC, Hayward RA. VADT Investigators. Intensive Glucose Control in Patients with Type 2 Diabetes - 15-Year Follow-up. N Engl J Med. 2019;380(23):2215-24.

32. Yang HK, Kang B, Lee SH, Yoon KH, Hwang BH, Chang K, Han K, Kang G, Cho JH. Association between hemoglobin A1c variability and subclinical coronary atherosclerosis in subjects with type 2 diabetes. J Diabetes Complications. 2015;29(6):776-82.

33. Intensive blood-glucose. control with sulphonylureas or insulin compared with conventional treatment and risk of complications in patients with type 2 diabetes (UKPDS 33). UK Prospective Diabetes Study (UKPDS) Group. Lancet. 1998;352(9131):837-53.

34. Gordon DJ, Probstfield JL, Garrison RJ, Neaton JD, Castelli WP, Knoke JD, Jacobs DR Jr, Bangdiwala S, Tyroler HA. High-density lipoprotein cholesterol and cardiovascular disease. Four prospective American studies. Circulation. 1989;79(1):8-15.

35. Barter P, Gotto AM, LaRosa JC, Maroni J, Szarek M, Grundy SM, Kastelein JJ, Bittner V, Fruchart JC. Treating to New Targets Investigators. HDL cholesterol, very low levels of LDL cholesterol, and cardiovascular events. N Engl J Med. 2007;357(13):1301-10.

36. van Capelleveen JC, Bochem AE, Boekholdt SM, Mora S, Hoogeveen RC, Ballantyne CM, Ridker PM, Sun W, Barter PJ, Tall AR, et al. Association of High-Density Lipoprotein-Cholesterol Versus Apolipoprotein A-I With Risk of Coronary Heart Disease: The European Prospective Investigation Into Cancer-Norfolk Prospective Population Study, the Atherosclerosis Risk in Communities Study, and the Women's Health Study. J Am Heart Assoc. 2017;6(8):e006636.

37. Riwanto $M$, Landmesser $U$. High density lipoproteins and endothelial functions: mechanistic insights and alterations in cardiovascular disease. J Lipid Res. 2013;54(12):3227-43.

38. Creager MA, Lüscher TF, Cosentino F, Beckman JA. Diabetes and vascular disease: pathophysiology, clinical consequences, and medical therapy: Part I. Circulation. 2003;108(12):1527-32. 
39. Bobbert T, Mai K, Fischer-Rosinsky A, Pfeiffer AF, Spranger J. A1C is associated with intima-media thickness in individuals with normal glucose tolerance. Diabetes Care. 2010;33(1):203-4.

\section{Figures}

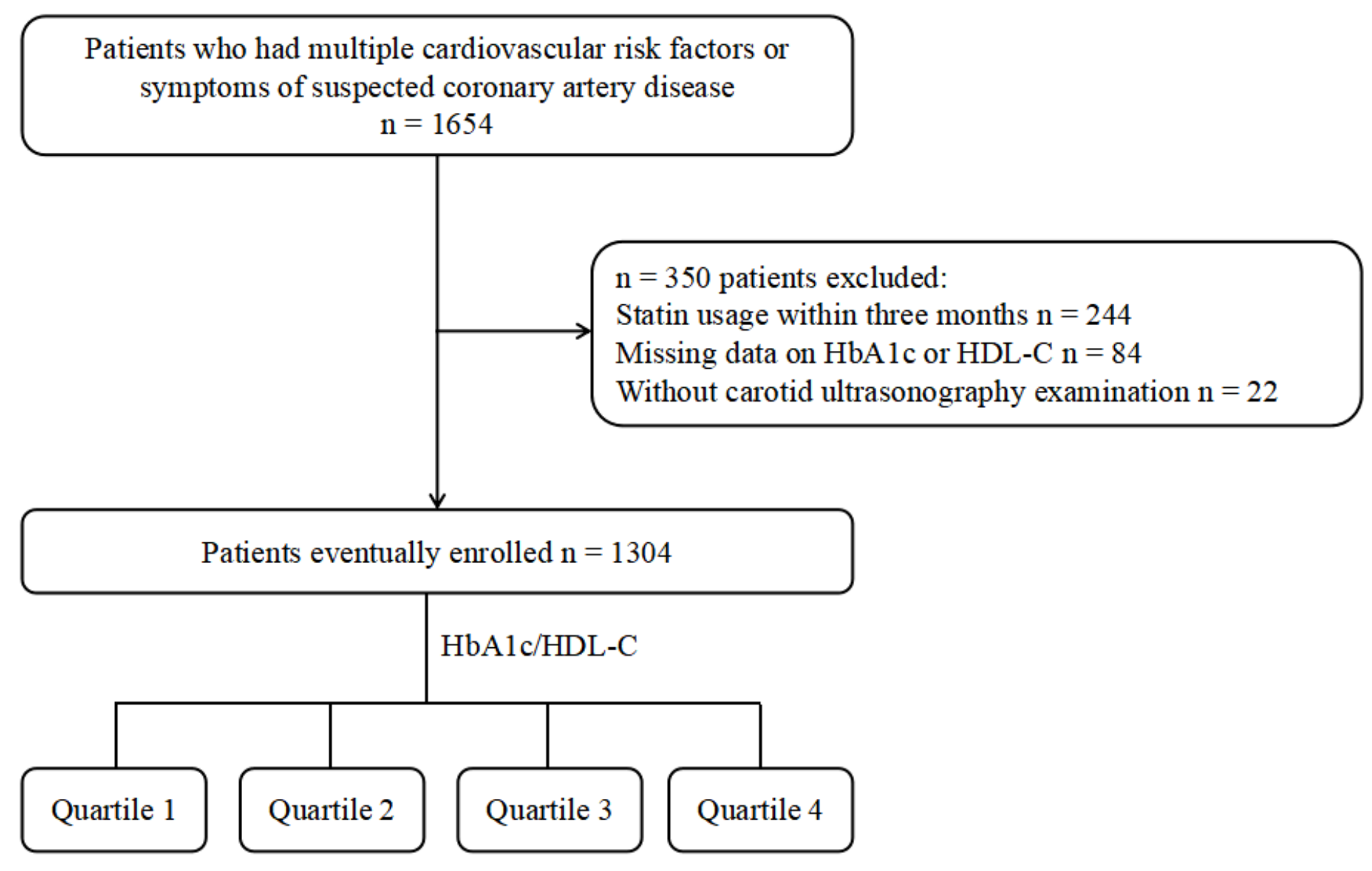

Figure 1

Flow chart.
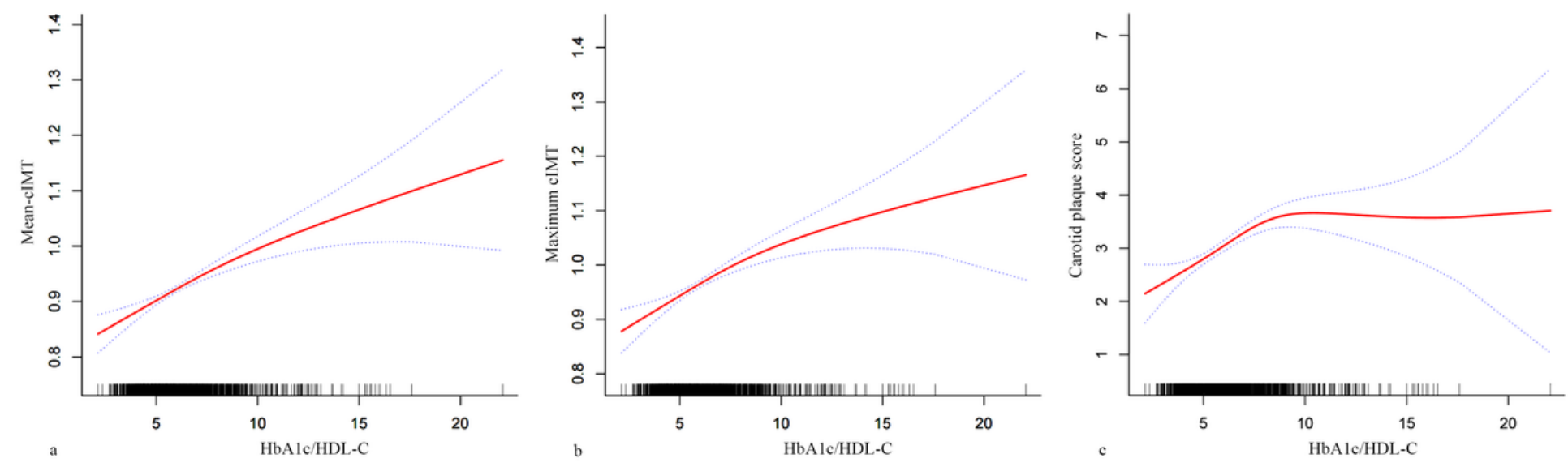

Figure 2 
Dose-response relationship between $\mathrm{HbA} 1 \mathrm{c} / \mathrm{HDL}-\mathrm{C}$ ratio and $\mathrm{cIMT}$ in patients with high-risk CVD. The area between the two dotted lines represents the $95 \%$ confidence interval. a) Mean cIMT (P for trend: $<0.001)$; b) maximum cIMT (P for linear trend: $<0.001)$; $c$ ) carotid plaque score ( $P$ for trend: $<0.001$ ). $\mathrm{HbA1c}$ hemoglobin Alc, HDL-C high-density lipoprotein cholesterol, cIMT carotid intima-media thickness, CVD cardiovascular diseases

All patients
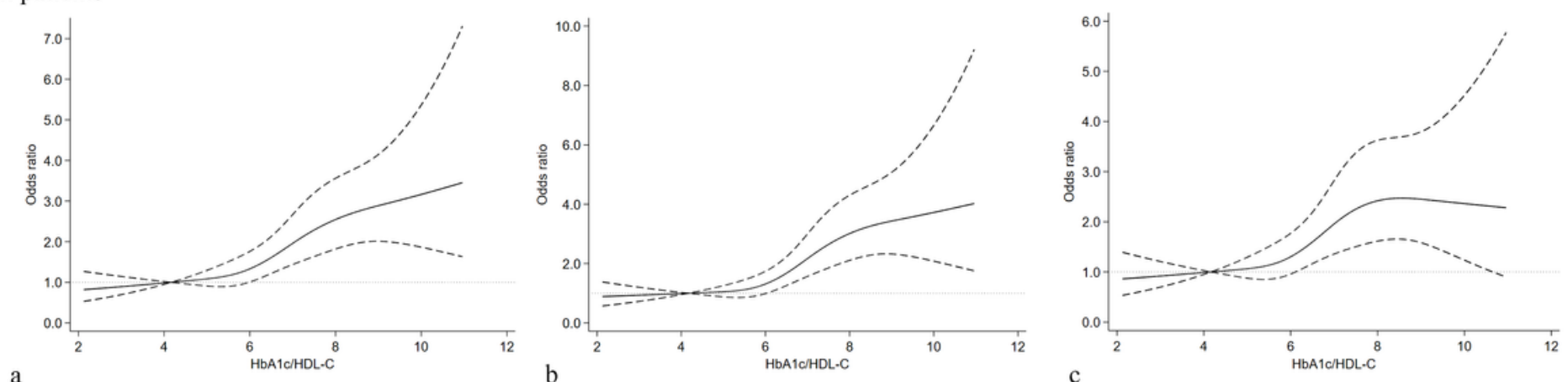

DM

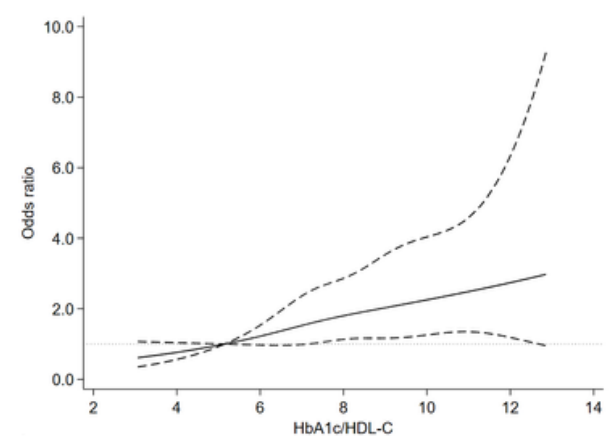

d

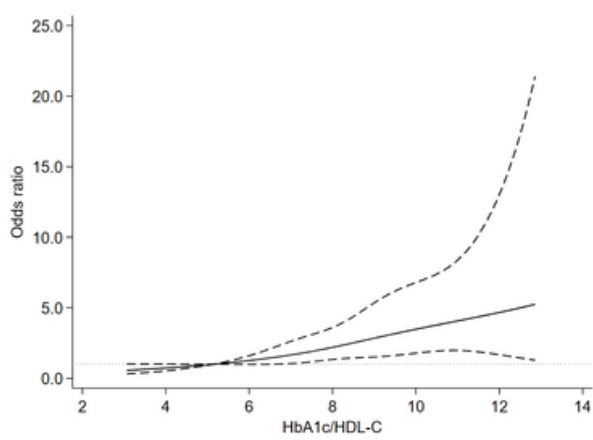

e

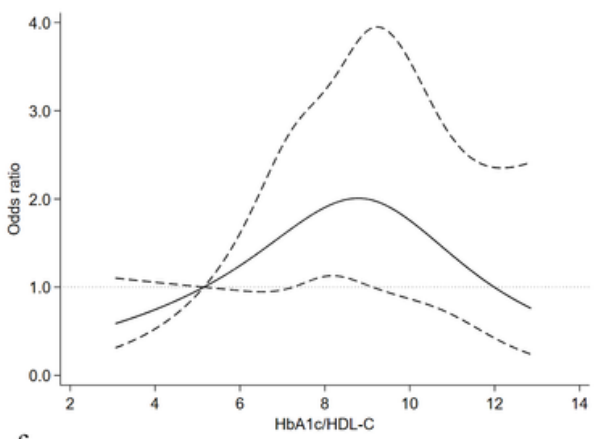

f

non-DM
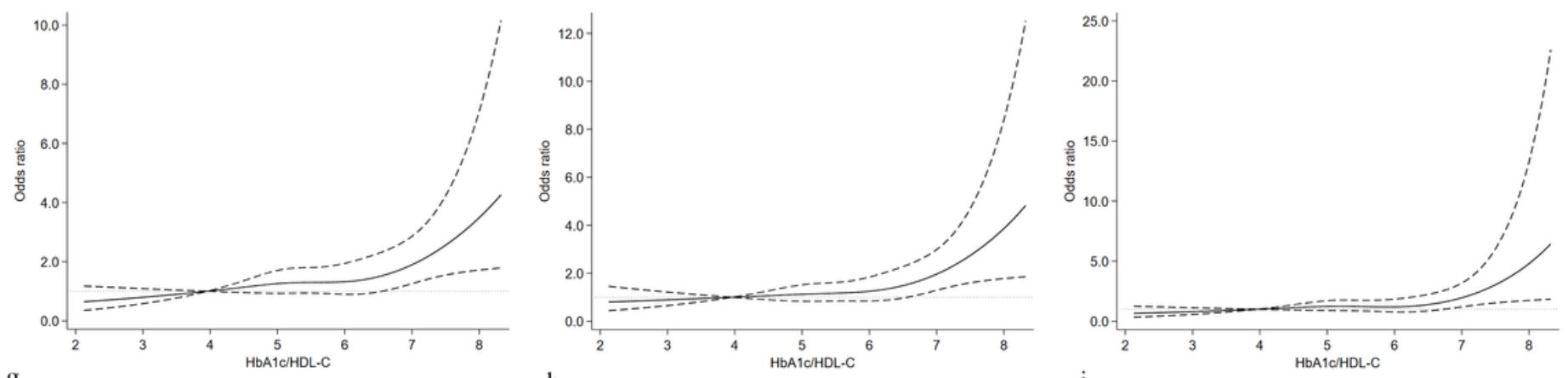

\section{Figure 3}

Dose-response relationship between $\mathrm{HbA1} / \mathrm{CDL}-\mathrm{C}$ ratio and carotid atherosclerosis. $\mathrm{a}, \mathrm{b}, \mathrm{c}$ ) Abnormal mean cIMT; d, e, f) abnormal maximum cIMT; $g, h$, i) carotid artery plaque. Note: Odds ratios and $95 \%$ confidence intervals (Cls) were adjusted for the same variables as in Model 4 (Table 2). The reference value of the $\mathrm{HbA} 1 \mathrm{c} / \mathrm{HDL}-\mathrm{C}$ ratio is the median of the reference group. The area between the two dotted lines represents the $95 \% \mathrm{Cl}$. Owing to the small sample and large $95 \% \mathrm{Cl}$, the highest $4 \%$ of patients are not shown in the figure. HbA1c hemoglobin Alc, HDL-C high-density lipoprotein cholesterol, cIMT carotid intima-media thickness, DM diabetes mellitus 


\section{Supplementary Files}

This is a list of supplementary files associated with this preprint. Click to download.

- Additionalfile1.doc 